\title{
Implementation of Modified Harris Corner Detector Algorithm -Including Free Parameters Based on FPGA
}

\author{
Chandaka Shravani, Rudra Pratap Das
}

\begin{abstract}
An efficient, pipelined Field Programmable Gate Arrays (FPGA) engineering of a modified Harris corner Detector is proposed. In laptop imaginative and prescient, the Harris nook encompass locator is one of the most fundamental strides in numerous precious applications, as an instance, three-D replica. in any case, inside the occasion that it's miles actualized in programming, the following code is not affordable to be achieved continuously by using minimum attempt versatile processors. device technique has been acquired for offloading the monotonous element extraction method into reason entryways with the purpose that the association is having minimal attempt to supply and low capability to paintings contrasted with its product accomplice. The framework is fabricated and attempted on a field programmable Gate Arrays(FPGA) level (Zed board). The assessments and demos exhibit that the speed and precision of the component indicator are enough for some proper applications. The results reveal an ideal concord between belongings utilization and timing execution, contrasted and previous.
\end{abstract}

Index Terms-Field Programmable Gate Arrays, Computer Vision, Harris Corner detector

\section{INTRODUCTION}

Constantly superior sign packages area programmable entryway show off (FPGA) were present as one in all applied in the system field of automated indicators, utilising parallel coping with. previous due to computational unpredictability a giant lot of calculations had been walking with the decrease rate. In FPGA it's far even attainable to cope with the non directly qualities. In various software areas of reconfigurable gadgets, FPGAs have broadened their application into image managing circle as nicely. image getting ready activities are fundamentally of two kinds: low stage advertisement unusual state. in this paintings we are involved about the low stage photo making ready sports consisting of spotlight recognition. It includes first pastime on a photo, by inspecting every unmarried pixel to test whether or not there is an element gift at that pixel. A function is a hallmark a few portion of a picture like edges, corners, hundreds, edges. Corners focuses can be characterised as pixel wherein surprising change in force occur. those have excessive bend and lie in the locale among edges. nook recognition includes low level photograph handling precious for laptop imaginative and prescient packages like movement following [1], stereo vision [2], movement following [3].

Revised Manuscript Received on April 12, 2019.

Chandaka Shravani, Assistant Professor, Vignan's Institute of information Technology, AP, India.

Rudra Pratap Das, Assistant Professor, Vignan's Institute of information Technology, AP, India
Extensive assortment of fashions are located in implanted making ready like decreased education set computing(RISC), digital sign processors(DSP), application express incorporated Circuit(ASIC), application precise instruction set processor(ASIP), field Programmable Gate Array(FPGA), and Programmable system on Chip(SoC). every engineering has its own favorable situations and weaknesses. Miniaturized scale programmable gadgets are chosen in step with the specific necessity. FPGAs are broadly applied as a result of their parallelism, adaptability and short time to be useful in marketplace. The fundamental fee of FPGAs is that their programmable additives can be designed to have required usefulness. besides FPGAs accomplish advanced than GPUs and DSPs [4] as a ways as energy and timing execution.

\section{ASSOCIATED PAINTINGS}

Many nook finders were mentioned up till now. essentially two lessons exist. On eis shape based totally and the alternative is electricity based totally. form based totally techniques to begin with recoup picture shapes and after that quest for ebb and flow maxima or expression focuses along those forms. The number one task for automatic photograph acknowledgment became the shading based totally calculation (shading histogram or shading distributive highlights). Shading histogram become fruitful and faster in distinguishing shading conveyance includes in a few random images assembly vital necessities. besides it become useless in coordinating big arrangement of snap shots and did now not satisfy the accompanying standards (Consistency, Accuracy) [5]. The later endeavors have been restricted to the distinguishing proof of corners and edges. Harris corner Detector which fused crafted by Harris and Stephen fundamental work. Harris become powerful in distinguishing sturdy highlights in a few random image. because it was just figuring out corners, his work is absence of community of spotlight focuses which had obstacles in getting full-size descriptors, as an example, surfaces and articles. Later some other nook finder calculation called speedy (features from accelerated phase take a look at) changed into exhibited. The 0.33 enterprise includes image coordinating from finished photo with jumbled foundations. it's miles applicable to realize that element based calculations have been broadly applied as highlight point locators as hues, corners and edges relate to picture 
colorations and areas individually. excessive statistics that these may be coordinated with snap shots. The issue primarily based locators possibly carry out exactly while the articles to be coordinated have an equal shading or an unmistakable nook or aspect. besides, the thing prepare calculations do paintings with respect to in situations in which photographs are uncovered to types in shading's conveyance, scale, mild, turn or relative exchange. some other magnificence of picture coordinating calculation changed into grown almost inside the timeframe. these calculations are referred to as surface primarily based calculations because of their capacity to in shape includes between diverse images notwithstanding the nearness of finished foundations and lack of planar and nicelycharacterized edges. An epic technique changed into embraced through David Lowe. Lowe [12] who exhibited SIFT (Scale Invariant feature rework) for keeping apart clean highlights from pics which haven't any range for photograph scale and pivot. that is applied in severa packages contain picture mosaic, acknowledgment, restoration and so on. After Lowe, Ke and Sukthankar applied PCA (primary issue analysis-SIFT) to standardize slope repair in place of histograms. They demonstrated that PCA-based nearby descriptors had been moreover precise and compelling to picture distortions. The strategies for extricating hearty highlights were nonetheless extremely moderate. Cove et al. SURF (speeded up hearty highlights) [13] and applied important photographs for photo convolutions in speedy-Hessian indicator. successful and proficient reputation and portrayal of spotlight focuses is a miles considered trouble in laptop vision. The place of spotlight focuses may be finished in situations where a close-by picture window is slided over the whole photo to apprehend corners as highlight focuses. With every slide the window is moved in one-of-a-kind approaches to decide modifications within the regular pixel force. a few changes to this technique were proposed. one-of-a-kind calculations have been proposed to construct unmistakable descriptors for outstanding corners. spotlight extraction reachable on unmarried chip is a useful usage for the laptop imaginative and prescient packages.

The paper changed into sorted out as, section I gives the importance of low degree picture responsibilities in their execution on chip, phase II associated work and section III approach for utilization of MHCD on FPGA, vicinity IV Derivation of modified Harris corner Detector calculation phase $\mathrm{V}$ consequences segment VI give up.

\section{METHOD}

Low level factor extraction strategies get valuable facts from the photograph facts for the laptop vision application. here on this paper corners are removed from the practise facts. The maximum generally utilized calculation for corner extraction is Harris nook identifier calculation but it has its very very own downsides like rotational invariant and obstacle problems. In HCD calculation the response condition is given by way of,

$$
\left.R=\operatorname{det}(X)-k(\operatorname{trace}(X))^{2}\right)
$$

Where, ok is an exactly selected well worth and its in reality well worth reaches from zero.04 to 0.25 . Better preference of good enough honestly well worth gives splendid effects and it changed into confirmed and scaled so. Be that as it is able to, chose the quality incentive for $\mathrm{k}$ devours a part of time through trying out the statistics greater than as soon as.

Right here on this paper we've notion of a changed Harris nook Detector calculation that demonstrates that experimental capabilities won't pass judgment on the calculation execution and with the aid of utilising fake neural systems for the coaching information the yield results can be upgraded.

Ventures to gather nook focuses the use of MHCD calculation:

1. Acquire separation.

2. Go with the flow a roundabout Gaussian window over the picture and discover the convolution Matrices for the two subsidiaries $\mathrm{dx}$ and dy as Ix and Iy.

3. Shape the Harris community $\mathrm{X}$

4. Calculate cornerness of each pixel from the Harris network. Making use of the adjusted HCD calculation.

5. Exercise non maximal concealment.

6. Observe neural based totally calculations to beautify the photo nice and desire.

Execution in FPGA and growing IP:

The rectangular chart for execution of previously stated calculation into FPGA is given in decide 1,

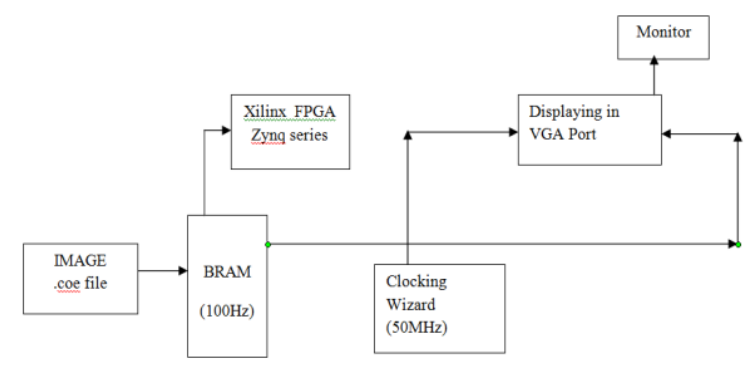

Figure 1: Schematic for FPGA Implementation

VGA port of Xilinx FPGA Zynq association has signals HS, VS, R, G, B. The photo file must be shown on the processor have to be within the .coe function. next to stacking the streamlined image facts at the BRAM, the timing wizard IP will isolate the clock recurrence of BRAM from $100 \mathrm{MHz}$ to $50 \mathrm{Mhz}$ a good way to synchronize with the VGA port. The Board being applied IP squares need to be covered for each plan in Block design utilization of Vivado HLS. HS and VS are the counters to guide clock from 100MHzto 50MHz.

\section{Stepped forward HCD calculation:}

Most of the calculations that are applied for highlight extraction Harris corner Detector is the easiest and most commonly utilized calculation. it's far at the beginning proposed by way of Moravec [7] and later stepped forward by Harris and Stephens to channel commotion making use of Gaussian window rather than square window.

Harris acquires the corners through utilizing Eigen esteems and the Harris lattice is given through 


$$
X=\begin{array}{cc}
I_{x}^{2} & I_{x} I_{y} \\
I_{y} I_{x} & I_{y}^{2}
\end{array}
$$

The matrix can be obtained by calculating the derivatives of image $\mathrm{I}$ in both $\mathrm{u}$ and $\mathrm{v}$ directions on a circular Gaussian window.

Here $\mathrm{X}$ is the autocorrelation matrix of the target pixel, $\lambda 1$ and $\lambda 2$ are the eigen values.

If $\lambda 1$ and $\lambda 2<<0$ then the area near the target pixel is flat area,

$\lambda 1>\lambda 2$ or $\lambda 1$ or $\lambda 2$ then the region is an edge

$\lambda 1$ and $\lambda 2 \gg 0$ then the region indicates there is a drastic change in the intensity value, this is called as a corner.

Where $\operatorname{det}(\mathrm{X})=\lambda 1 \lambda 2$ (determinant) $\mathrm{X}$, trace $(\mathrm{X})=$ $\lambda 1+\lambda 2$ (trace) of the matrix.

The value of $\mathrm{k}$ ranges from 0.04 to 0.15 and it is an empirically selected constant value.

When $\mathrm{R}$ value is greater than the threshold then the pixel is considered as a corner.

The response function has to obey the condition $\lambda 1$ and $\lambda 2 \gg 0$ but in the interval of [0 1$]$ to obtain the corners.

Let $\mathrm{n}=\lambda 1 / \lambda 2 \geq 1$

The response equation (1) is given by,

$\left.R=\operatorname{det}(X)-k(\operatorname{trace}(X))^{2}\right)>$ threshold

The Eigen ratio is given by

$E r=(\lambda 1+\lambda 2)^{2} / \lambda 1 \lambda 2$

$E r=(n \lambda 2+\lambda 2)^{2} /(n \lambda 2)^{2}=(n+1)^{2} / n=n+2+n^{-1}$

Now the response function is modified as,

$R=\operatorname{det}(A)\left(1-\left(\operatorname{trace}(A)^{2} / \operatorname{det}(A)\right)\right.$

$R=\operatorname{det}(A)\left(1-\left(n+2+n^{-1}\right) / \operatorname{det}(A)\right.$

For, $\mathrm{n}=0.2$

$$
\begin{aligned}
& R=\operatorname{det}(\mathrm{X}) \times(1-4.5 \times 0.1)=\operatorname{det}(X) \times 0.55 \\
& \quad \Rightarrow R \text { corresponds to intensity corner. }
\end{aligned}
$$

For, $\mathrm{n}=100$ i.e a larger value $\mathrm{R}$ corresponds to -9.2 giving an edge.

For different $\mathrm{n}$ and the corresponding computed value of $\mathrm{R}$ indicates corner or edge. The test results proved theoretically that the values of $\mathrm{n}$ ranges from 0.04 to 0.06 .

Basically, $(\lambda 1+\lambda 2)$ Is a proportion of power as a protracted manner as comply with which simply smothers the edges. The event of corner terms region where at least edges meet. For degree areas there is no adjustment each which manner, for edges there may be no adjustment in pressure in side bearing. In which concerning corners there is a huge alternate each which way.

$n$ upper limit:

We can't write $\lambda 1$ and $\lambda 2-$

$$
\mathrm{n}(\lambda 1+\lambda 2)^{2}=\lambda_{1}^{2}\left(\beta-n(1+\beta)^{2}\right)
$$

Where, $\beta=\lambda 2 / \lambda 1$

For this term to be positive,

$0 \leq \mathrm{n} \leq \beta /(1+\beta)^{2} \leq 0.25$

For small $\beta$, the term becomes $\lambda_{1}^{2}(\beta-n), n \ll \beta$
The value of $\mathrm{n}$ has to be lower than 0.25 . The preferred value of $\mathrm{n}$ are in the range of 0.04 to 0.06 .

\section{RESULTS}

The altered Harris corner Detector calculation is mounted to the check images and actualized in MATLAB. Check picture is attached for the Harris nook finder and were given the corner point and a similar picture is hooked up for the MHCD with out making use of the free parameters and will acquire better consequences and regularly style of corner focuses. The detail focuses received for the normal Harris corner locator and the MHCD are seemed in determine 2 and figure 3 beneath for diverse test photographs.

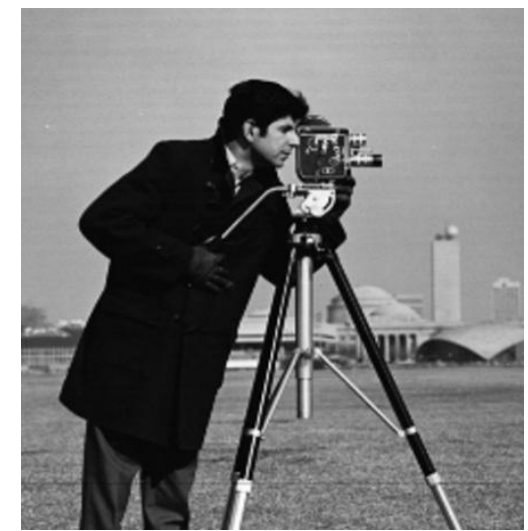

(a)

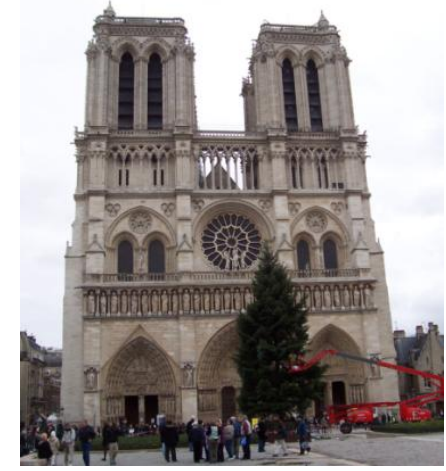

(b)

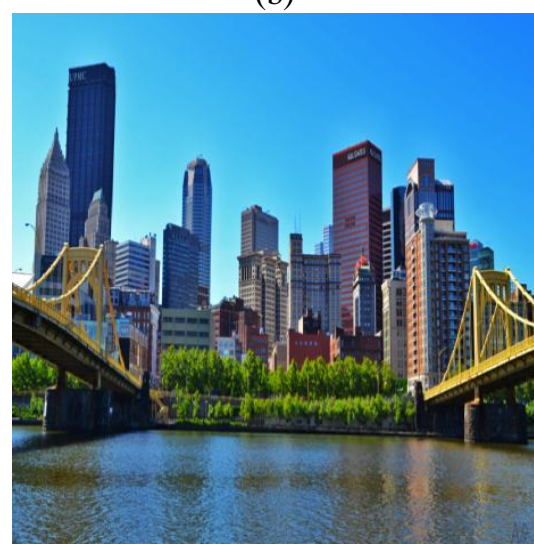

(c)

Figure 2.(a) Gray scale cameraman (b) RGB Notre Dame (c) RGB Pittsburg input images 


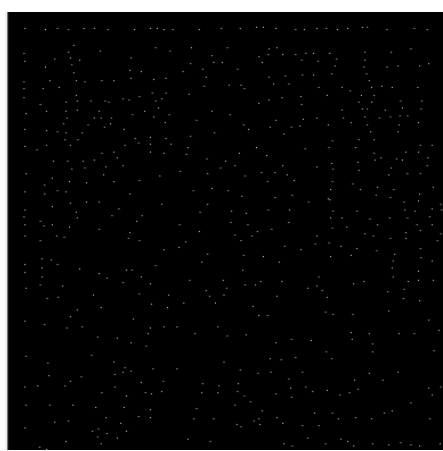

(d)

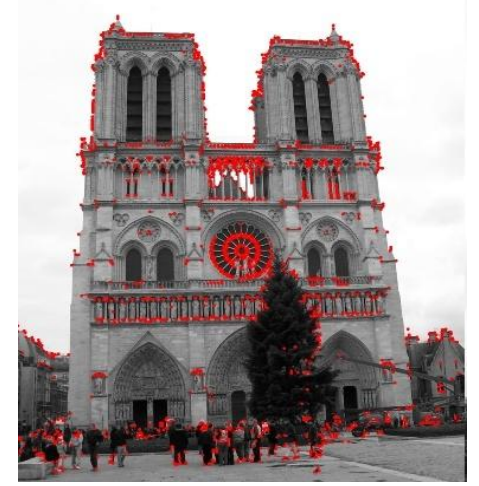

(e)

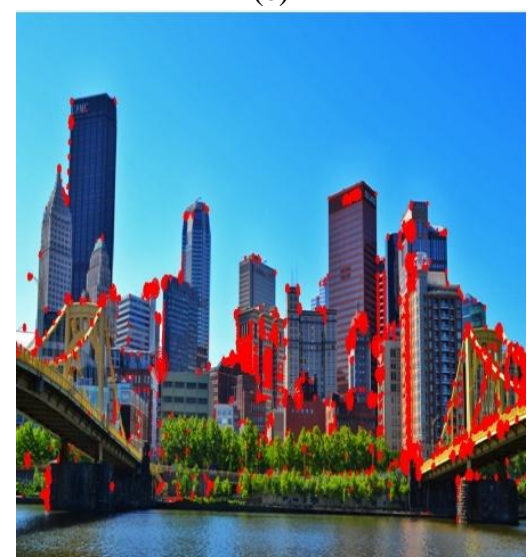

(f)

Figure 3. Corners obtained for the three input images (a), (b),(c) respective (d),(e),(f) using MHCD

The attempted pix are related to the line cushions and actualized on Zed board. The planning reaction and the asset use rundown for the execution is as seemed in table1. From the acquired outcomes, proposed MHCD may additionally want to present proficient utilization concerning property and timing examination whilst contrasted with the first Harris nook Detector.

\begin{tabular}{|l|c|c|c|c|}
\hline $\begin{array}{c}\text { Input } \\
\text { Image }\end{array}$ & Registers & Memory(kb) & LUTs & $\begin{array}{c}\text { Time } \\
(\mathrm{ms})\end{array}$ \\
\hline (a) & 5034 & 312 & 5846 & 4.15 \\
\hline (b) & 6348 & 452 & 6461 & 4.25 \\
\hline (c) & 15246 & 954 & 7354 & 4.29 \\
\hline
\end{tabular}

Table I. Resource Utilization and timing summary

\section{CONCLUSION}

As a element of improvement of effective FPGA based totally Harris corner locator, the essential calculations were checked. The Eigen percentage has been accentuated. Its breaking elements were checked in that tolerating the experimental well worth stated by means of the professionals. Streamlined numerical connection have been worked at for this reason. With the improved elements next purpose is to affirm the calculation the usage of ANFIS. The upsides of ANFIS in time period of facet identification joined with the advanced parameters. Guarantee that the FPGA utilization is upgraded..

\section{REFERENCES}

1. Kung, Ying-Shieh, and Gua-Shieh Shu. "development of a FPGA-based movement manipulate IC for robot arm." In business era, 2005. ICIT 2005. IEEE international convention on, pp. 1397-1402. IEEE, 2005.

2. Jin, Seunghun, Junguk Cho, Xuan Dai Pham, Kyoung Mu Lee, Sung-Kee Park, Munsang Kim, and Jae Wook Jeon. "FPGA form and utilization of a non-stop stereo vision framework." IEEE exchanges on circuits and frameworks for video innovation 20, no. 1 (2010): 15-26.

3. Black, Michael J., and Yaser Yacoob. "Following and perceiving unbending and non-rigid facial actions utilizing close by parametric models of image movement." In computer imaginative and prescient, 1995. Processes., 5th worldwide conference on, pp. 374-381. IEEE, 1995.

4. Che, Shuai, Jie Li, Jeremy W. Sheaffer, Kevin Skadron, and John Lach. "Quickening take a look at in focused applications with GPUs and FPGAs." In software particular Processors, 2008. SASP 2008. Symposium on, pp. One hundred and one107. IEEE, 2008.

5. Tieu, Kinh, and Paul Viola. "Boosting image recovery." worldwide magazine of computer imaginative and prescient 56, no. 1-2 (2004): 17-36.

6. El-Gayar, M. M., and H. Soliman. "A relative studies of picture low degree aspect extraction calculations." Egyptian Informatics magazine 14, no. 2 (2013): 175-181.

7. Moravec, Hans P. Snag evasion and path in reality through a seeing robot wanderer. No. STAN-CS-80-813. STANFORD UNIV CA DEPT OF laptop technological expertise, 1980.

8. R. Szeliski, laptop imaginative and prescient: calculations and programs: Springer era and business enterprise Media, 20 I O.

9. M. Trajkovi6 and M. Hedley, "brief nook vicinity," image and imaginative and prescient processing. Vol. Sixteen, pp. 75-87, 1998.

10. C. Harris and M. Stephens, "A joined comer and aspect indicator," in Alvey imaginative and prescient meeting, 1988, p. 50.

11. P. Possa, S. Mahmoudi, N. Harb, C. Valderrama, and P. Manneback, "A multi-goals fj:Jga-primarily based engineering for non-stop factor and comer identification," 2013.

12. Lowe, D.G.: unique image highlights from scale-invariant keypoints. Int. J. Comput. Vis.60(2), ninety one-one hundred ten (2004).

13. Bay, H., Ess, A., Tuytelaars, T., Gool, L.: Speeded-up lively highlights (SURF). Comput. Vis.Picture Underst. A hundred and ten(three), 346-359 (2008) 


\section{AUTHORS}

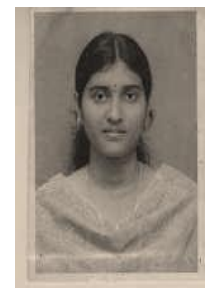

Ch.Shravani completed her M.tech and doing her Ph.D in VLSI Architectures. Working in Vignan's Institute of information Technology as Assistant Professor. Her Areas of interest are VLSI, Image processing.

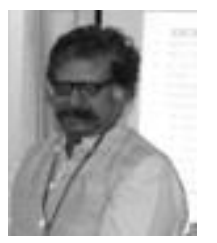

Rudra Pratap Das, Ph.D, Senior IEEE Member, Senior Professor, Vignan's Institute of information Technology(A). His areas of research are Fuzzy Logic, Signal Processing, Artificial Neural Networks, Image Processing. 